Pacific Journal of Mathematic 


\title{
UNITARY EQUIVALENCE TO INTEGRAL OPERATORS
}

\author{
V. S. SUNDER
}

A bounded operator $A$ on $L^{2}(X)$ is called an integral operator if there exists a measurable function $k$ on $X \times X$ such that, for each $f$ in $L^{2}(X)$,

and

$$
\int|k(x, y) f(y)| d \mu(y)<\infty \quad \text { a.e. }
$$

$$
A f(x)=\int k(x, y) f(y) d \mu(y) \text { a.e. }
$$

(Throughout this paper, $(X, \mu)$ will denote a separable, $\sigma$-finite measure space which is not purely atomic.) An integral operator is called a Carleman operator if the inducing kernel $k$ satisfies the stronger requirement:

$$
\int|k(x, y)|^{2} d \mu(y)<\infty \text { for almost every } x \text { in } X \text {. }
$$

In a recent paper ([2]), V. B. Korotkov characterized those operators that are unitary equivalent to operators $A$ on $L^{2}(X)$ such that both $A$ and $A^{*}$ are Carleman operators. It is natural to consider the same question with "Carleman" replaced by "integral." This question is settled by Theorem 2 .

An integral operator is absolutely bounded if the representing kernel $k$ is such that $|k|$ also induces a bounded integral operator. Theorem 3 furnishes three-fourths of a characterization of those operators that are unitarily equivalent to absolutely bounded integral operators. To be more specific, that theorem yields a necessary condition which is shown to be sufficient under an extra assumption. It is, however, the author's belief that this extra requirement is met by every bounded operator on a separable Hilbert space. This belief is formally stated as a conjecture at the end of the paper.

Lemma 1. Let $A$ be a bounded operator on a separable Hilbert space $\mathscr{K}$. The following conditions on $A$ are equivalent:

(i) There exists a Hilbert-Schmidt operator $K$ such that $\operatorname{ker}(A-K) \cap \operatorname{ker}\left(A^{*}-K^{*}\right)$ is an infinite-dimensional subspace of $\mathscr{H}$;

(ii) There exists an orthonormal sequence $\left\{e_{n}\right\}_{n=1}^{\infty}$ in $\mathscr{H}$ such that $\left\|A e_{n}\right\| \rightarrow 0$ and $\left\|A^{*} e_{n}\right\| \rightarrow 0$;

(iii) 0 belongs to the essential spectrum of $A^{*} A+A A^{*}$.

Proof. The implication (i) $\Rightarrow$ (ii) is proved by picking an orthonormal sequence $\left\{e_{n}\right\}_{n=1}^{\infty}$ in $\operatorname{ker}(A-K) \cap \operatorname{ker}\left(A^{*}-K^{*}\right)$ and recalling that $K$ is compact. 
(ii) $\Rightarrow$ (iii). Pick a subsequence, if necessary, and assume (without loss of generality) that $\sum_{n}\left(\left\|A e_{n}\right\|+\left\|A^{*} e_{n}\right\|\right)<\infty$. Since

$$
\begin{aligned}
\left\|\left(A^{*} A+A A^{*}\right) e_{n}\right\| & \leqq\left\|A^{*}\right\|\left\|A e_{n}\right\|+\|A\|\left\|A^{*} e_{n}\right\| \\
& =\|A\| \quad\left(\left\|A e_{n}\right\|+\left\|A^{*} e_{n}\right\|\right),
\end{aligned}
$$

it follows that $\sum_{n}\left\|\left(A^{*} A+A A^{*}\right) e_{n}\right\|<\infty$.

So, if $P$ denotes the projection onto $\bigvee\left\{e_{n}\right\}_{n=1}^{\infty}$, the operator $\left(A^{*} A+A A^{*}\right) P$ is compact. Since $A^{*} A+A A^{*}$ is a compact perturbation of $\left(A^{*} A+A A^{*}\right)(1-P)$, and since $\left(A^{*} A+A A^{*}\right)(1-P)$ has an infinite-dimensional kernel (containing $\operatorname{ran} P$ ), it is clear that $0 \in \sigma_{e}\left(A^{*} A+A A^{*}\right)$.

(iii) $\Rightarrow$ (i). If $0 \in \sigma_{e}\left(A^{*} A+A A^{*}\right)$, there exists an orthonormal sequence $\left\{\varphi_{n}\right\}_{n=1}^{\infty}$ such that $\left\|\left(A^{*} A+A A^{*}\right) \varphi_{n}\right\|<1 / 2^{n}$. If $\left\{\varphi_{n}\right\}_{n=1}^{\infty}$ is extended to an orthonormal basis $\left\{\psi_{n}\right\}_{n=1}^{\infty}$ of $\mathscr{H}$ and if $E$ denotes the projection onto $\mathrm{V}\left\{\varphi_{n}\right\}_{n=1}^{\infty}$, it follows that

$$
\begin{aligned}
\sum_{n}\left(\left\|A E \psi_{n}\right\|^{2}+\left\|A^{*} E \psi_{n}\right\|^{2}\right) & =\sum_{n}\left(\left\|A \varphi_{n}\right\|^{2}+\left\|A^{*} \varphi_{n}\right\|^{2}\right) \\
& =\sum_{n}\left(\left(A^{*} A+A A^{*}\right) \varphi_{n}, \varphi_{n}\right) \\
& \leqq \sum_{n}\left\|\left(A^{*} A+A A^{*}\right) \varphi_{n}\right\| \\
& <\infty .
\end{aligned}
$$

Hence, both $A E$ and $A^{*} E$ are Hilbert-Schmidt operators. Since the collection of Hilbert-Schmidt operators on $\mathscr{H}$ is a self-adjoint twosided ideal of $\mathscr{B}(\mathscr{H})$, the operator $K=A E+E A(1-E)$ is also a Hilbert-Schmidt operator.

Observe that

$$
\begin{aligned}
A-K & =A-A E-E A(1-E) \\
& =(1-E) A(1-E)
\end{aligned}
$$

and conclude that $\operatorname{ker}(A-K) \cap \operatorname{ker}\left(A^{*}-K^{*}\right)$ contains the infinitedimensional subspace $\operatorname{ran} E$.

In view of the equivalence of (ii) and (iii), Korotkov's theorem on bi-Carleman operators can be stated thus:

"An operator $A$ on a separable Hilbert space is unitarily equivalent to an operator $T$ on $L^{2}(X)$ such that both $T$ and $T^{*}$ are Carleman operators if and only if $0 \in \sigma_{e}\left(A^{*} A+A A^{*}\right)$."

The next result is similar.

THeOREM 2. An operator $A$ on a separable Hilbert space is unitarily equivalent to an operator $T$ on $L^{2}(X)$ such that both $T$ and $T^{*}$ are integral operators if and only if $0 \in \sigma_{e}\left(A^{*} A+A A^{*}\right)$. 
Proof. Since the "if" half of the theorem follows immediately from Korotov's result, it suffices to prove that if $A$ and $A^{*}$ are integral operators on $L^{2}(X)$, then $0 \in \sigma_{e}\left(A^{*} A+A A^{*}\right)$.

In view of some measure-theoretic isomorphism theorems, there is no loss of generality in assuming that $\mu(X)<\infty$. It is known (cf. [4]) that in this case, any integral operator, when viewed as an operator from $L^{2}(X)$ into $L^{1}(X)$, is compact, that the collection of operators on $L^{2}(X)$ which are $L^{2} \rightarrow L^{1}$ compact (to use an ad-hoc symbol) is a right-ideal of $\mathscr{B}\left(L^{2}(X)\right)$, and that 0 belongs to the right essential spectrum of every such operator. The assumption that $A$ and $A^{*}$ are integral operators on $L^{2}(X)$ implies, via the last statement, that $A^{*} A+A A^{*}$ is $L^{2} \rightarrow L^{1}$ compact and that $0 \in \sigma_{r e}\left(A^{*} A+A A^{*}\right)=$ $\sigma_{e}\left(A^{*} A+A A^{*}\right)$.

Observe that if the underlying measure space is $Z^{+}(=\{1,2,3, \cdots\})$, equipped with counting measure, an absolutely bounded integral operator on $L^{2}\left(\boldsymbol{Z}^{+}\right)\left(=\ell^{2}\right)$ is just an operator on $\ell^{2}$ that is induced by a matrix $\left(\alpha_{i j}\right)$ such that $\left(\left|\alpha_{i j}\right|\right)$ also induces a bounded operator on $\ell^{2}$. Matrices with this property will be called absolutely bounded.

THEOREM 3. If an operator $A$ on a separable Hilbert space $\mathscr{H}$ is unitarily equivalent to an absolutely bounded integral operator on $L^{2}(X)$, then $0 \in \sigma_{e}\left(A^{*} A+A A^{*}\right)$. Conversely, if $0 \in \sigma_{e}\left(A^{*} A+A A^{*}\right)$ and if $A$ has an absolutely bounded matrix with respect to some orthonormal basis of $\mathscr{H}$, then $A$ is unitarily equivalent to an absolutely bounded integral operator on $L^{2}(X)$.

Proof. It is well-know (cf. [3] and [5]) that the adjoint of an absolutely bounded integral operator is also an absolutely bounded integral operator. So, if $A$ is unitarily equivalent to an absolutely bounded integral operator on $L^{2}(X)$, it follows from Theorem 2 that $0 \in \sigma_{e}\left(A^{*} A+A A^{*}\right)$.

Conversely, suppose that $A$ has an absolutely bounded matrix with respect to some basis of $\mathscr{H}$, and that $0 \in \sigma_{e}\left(A^{*} A+A A^{*}\right)$. Lemma 1 guarantees the existence of a Hilbert-Schmidt operator $K$ such that $\operatorname{ker}(A-K) \cap \operatorname{ker}\left(A^{*}-K^{*}\right)$ is infinite-dimensional. Since $K$ has an absolutely bounded matrix with respect to every basis of $\mathscr{P}$, and since every Hilbert-Schmidt operator on $L^{2}(X)$ is an absolutely bounded integral operator, we may (and do) assume that $\operatorname{ker} A \cap \operatorname{ker} A^{*}$ is infinite-dimensional.

Since $X$ is not purely atomic, there exists a subset $X_{0}$ of positive measure such that $X_{0}$ contains no atoms. The non-atomicity of $X_{0}$ implies the existence of a partition $X_{0}=X_{1} \cup X_{2}$ into disjoint sets of positive measure. Since $X_{1}$ and $X_{2}$ contain no atoms, both $L^{2}\left(X_{1}\right)$ and 
$L^{2}\left(X_{2}\right)$ are infinite-dimensional. Since ker $A \cap \operatorname{ker} A^{*}$ is infinite- dimensional, the operator $A$ is unitarily equivalent to an operator on $L_{2}(X)$ which has the form $A_{1} \oplus 0 \oplus 0$ in the decomposition $L^{2}(X)=L^{2}\left(X_{1}\right) \oplus$ $L^{2}\left(X_{2}\right) \oplus L^{2}\left(X-X_{0}\right)$. By incorporating a "half" of the first zerosummand into $A_{1}$, we may assume that $A_{1}$ has the form $B \oplus 0$ with respect to some decomposition of $L^{2}\left(X_{1}\right)$ into infinite-dimensional subspaces. Since $A_{1}=B \oplus 0$ is clearly unitarily equivalent to $A_{1} \oplus 0 \oplus 0$, it follows that $A_{1}$ has an absolutely bounded matrix with respect to a suitable orthonormal basis of $L^{2}\left(X_{1}\right)$. So, it suffices to show that $A_{1} \oplus 0$ is unitarily equivalent to an absolutely bounded integral operator on $L^{2}\left(X_{0}\right)$.

Let $\left\{\varphi_{n}\right\}_{n=1}^{\infty}$ be an orthonormal basis of $L^{2}\left(X_{1}\right)$ with respect to which $A_{1}$ has an absolutely bounded matrix, say $\left(\alpha_{i j}\right)$. (Thus, $\alpha_{i j}=$ $\left(A \varphi_{j}, \varphi_{i}\right)$.) Let $\left\{\psi_{n}\right\}_{n=1}^{\infty}$ be an orthonormal basis for $L^{2}\left(X_{2}\right)$. Since $X_{0}$ is non-atmic, there exists a partition $\left\{E_{n}\right\}_{n=1}^{\infty}$ of $X_{0}$ into pairwise disjoint subsets of positive measure. Let $f_{n}=\mu\left(E_{n}\right)^{-1 / 2} \chi_{E_{n}}$. The sequence $\left\{f_{n}\right\}_{n=1}^{\infty}$ is clearly orthonormal. Let $\left\{g_{n}\right\}_{n=1}^{\infty}$ be an orthonormal basis for $L^{2}\left(X_{0}\right) \ominus \mathrm{V}\left\{f_{n}\right\}_{n=1}^{\infty}$.

Let $U$ be the unitary operator on $L^{2}\left(X_{0}\right)$ such that $U f_{n}=\varphi_{n}$ and $U g_{n}=\psi_{n}$ for $n=1,2, \cdots$. Then, if $T=U^{*}(A \oplus 0) U$, it is clear that $\bigvee\left\{g_{n}\right\}_{n=1}^{\infty} \subseteq \operatorname{ker} T \cap \operatorname{ker} T^{*}$. A moment's thought and some routine verification shows that $T$ is the integral operator on $L^{2}\left(X_{0}\right)$ induced by the kernel

$$
k(x, y)=\sum_{m} \sum_{n} \frac{\alpha_{m n} \chi_{E_{m}}(x) \chi_{E_{n}}(y)}{\mu\left(E_{n}\right)^{1 / 2} \mu\left(E_{m}\right)^{1 / 2}} .
$$

Straightforward computations show that the kernel $k$ is absolutely bounded precisely when the matrix $\left(\alpha_{m_{n}}\right)$ is absolutely bounded. Since the matrix $\left(\alpha_{m n}\right)$ was chosen to be absolutely bounded, it follows that $T$ is an absolutely bounded integral operater on $L^{2}(X)$, and the proof is complete.

CoROllaRY 4. Let $N$ be a normal operator whose spectrum is contained in a rectifiable curve in the complex plane. Then $N$ is unitarily equivalent to an absolutely bounded integral operator on $L^{2}(X)$ if and only if $0 \in \sigma_{e}(A)$.

Proof. I. D. Berg has shown ([2]) that every such normal operator is the sum of a diagonalizable operator and a Hilbert-Schmidt operator. This means that $N$ has an absolutely bounded matrix with respect to at least one basis - the one which consists of eigenvector of the corresponding diagonalizable operator will do. Since $0 \in \sigma_{e}(N)$ if and only if $0 \in \sigma_{e}\left(N^{*} N+N N^{*}\right)$, the assertion follows from Theorem 3. 
Conjecture. Every operator on a separable Hilbert space has an absolutely bounded matrix with respect to some orthonormal basis of the underlying Hilbert space.

\section{REFERENCES}

1. I. D. Berg, An extension of the Weyl-von Neumann theorem to normal operators, Trans. Amer. Math. Soc., 160 (1971), 365-371.

2. V. B. Korotkov, The unitary equivalence of linear operators to integral operators, Math. Notes, 19 (1976), 364-368.

3. M. A. Krasnoselskii, P. P. Zabreiko, E. I. Putsylnik and P. E. Sbolevskii, Integral operators in spaces of summable functions, Moscow (1966).

4. V. S. Sunder, Characterization theorems for integral operators, Ph. D. dissertation, Indiana University, 1977.

5. A. C. Zaanen, Linear Analysis, New York, 1953.

Received January 5, 1978.

University of California at Santa Barbara

Santa Barbara, CA 93106 



\section{PACIFIC JOURNAL OF MATHEMATICS}

\section{EDITORS}

DONALD BABBITT (Managing Editor)

University of Galifornia

Los Angeles, California 90024

Hugo RossI

University of Utah

Salt Lake City, UT 84112

C. C. MOORE AND ANDREW OGG

University of California

Berkeley, CA 94720
J. DUGUNDJI

Department of Mathematics University of Southern California Los Angeles, California 90007

R. Finn AND J. Milgram Stanford University Stanford, California 94305

\section{ASSOCIATE EDITORS}

R. ARENS

E. F. BECKENBACH

B. H. NEUManN

F. WOLF

K. YOSHIDA

\section{SUPPORTING INSTITUTIONS}

UNIVERSITY OF ARIZONA

UNIVERSITY OF BRITISH COLUMBIA CALIFORNIA INSTITUTE OF TECHNOLOGY

UNIVERSITY OF CALIFORNIA

MONTANA STATE UNIVERSITY

UNIVERSITY OF NEVADA, RENO

NEW MEXICO STATE UNIVERSITY

OREGON STATE UNIVERSITY
UNIVERSITY OF OREGON

UNIVERSITY OF SOUTHERN CALIFONIA

STANFORD UNIVERSITY

UNIVERSITY OF HAWAII

UNIVERSITY OF TOKYO

UNIVERSITY OF UTAH

WASHINGTON STATE UNIVERSITY

UNIVERSITY OF WASHINGTON 


\section{Pacific Journal of Mathematics}

\section{Vol. 92, No. $1 \quad$ January, 1981}

Michael E. Adams and J. Sichler, Lattices with unique complementation . ....1

Walter Allegretto, Positive solutions and spectral properties of second order

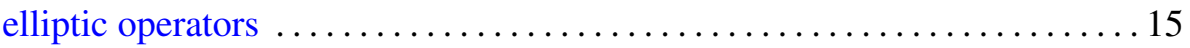

Philip J. Boland and Sean Dineen, Holomorphy on spaces of distribution . . 27

Duncan Alan Buell, Philip A. Leonard and Kenneth S. Williams, Note on

the quadratic character of a quadratic unit $\ldots \ldots \ldots \ldots \ldots \ldots \ldots \ldots \ldots$

Herbert Busemann and Bhalchandra B. Phadke, Two theorems on

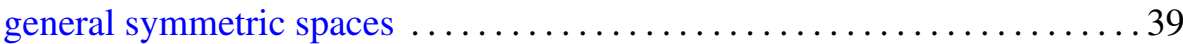

Emeric Deutsch, Bounds for the Perron root of a nonnegative irreducible

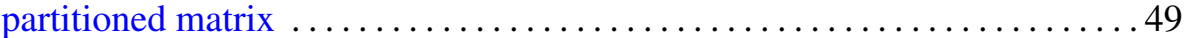

Charles F. Dunkl, A difference equation and Hahn polynomials in two

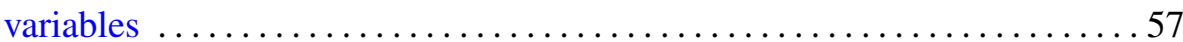

Gustave Adam Efroymson, The Riemann mapping theorem for planar Nash

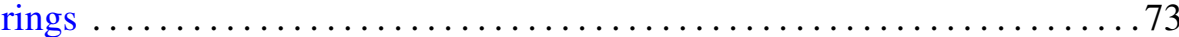

John Albert Fridy and Robert Ellis Powell, Tauberian theorems for matrices generated by analytic functions

Denton Elwood Hewgill, John Hamilton Reeder and Marvin Shinbrot,

Some exact solutions of the nonlinear problem of water waves .......887

Bessie Hershberger Kirkwood and Bernard Robert McDonald, The

symplectic group over a ring with one in its stable range

Esther Portnoy, Transitive groups of isometries on $H^{n}$

Jerry Ridenhour, On the sign of Green's functions for multipoint boundary

value problems

Nina M. Roy, An $M$-ideal characterization of $G$-spaces

Edward Barry Saff and Richard Steven Varga, On incomplete

polynomials. II

Takeyoshi Satō, The equations $\Delta u=P u(P \geqq 0)$ on Riemann surfaces and

isomorphisms between relative Hardy spaces

James Henry Schmerl, Correction to: "Peano models with many generic classes"

Charles Madison Stanton, On the closed ideals in $A(W)$

Viakalathur Shankar Sunder, Unitary equivalence to integral operators

Pavel G. Todorov, New explicit formulas for the $n$th derivative of composite functions

James Li-Ming Wang, Approximation by rational modules on boundary sets

Kenneth S. Williams, The class number of $Q(\sqrt{p})$ modulo 4 , for $p \equiv 5$

$(\bmod 8)$ a prime 